\title{
The transformation of chiral signals into macroscopic properties of materials using chirality-responsive polymers
}

\author{
Guangyan Qing ${ }^{1}$ and Taolei Sun ${ }^{1,2,3}$
}

The ability to transform the chiral signals of molecules into the macroscopic properties of a material will offer significant advantages in the development of chiral functional devices and chirality-related applications. Chirality-responsive polymers provide an excellent platform to realize this objective, which often involves two basic strategies. The first strategy is to utilize various external stimuli to directly mediate the chiral conformations of a polymer, through which the energy input is transformed into a macroscopic change in the properties of a material. The second strategy is to utilize the enantioselective interaction between polymers and guest chiral molecules to trigger a stepwise conformational change in smart polymers, which then results in transformation of the macroscopic properties. This review summarizes recent progress in generating chirality-responsive polymers based on these strategies and discusses advances in their applications as chiral sensors, liquid crystals, optical and electrical devices, nanomachines and so on. We then introduce the emerging field of chiral bio-interface materials, in which chiral signals are transformed into changes in the macroscopic behavior of cells and biomacromolecules based on the stereospecific interactions between biological systems and artificial materials.

NPG Asia Materials (2012) 4, e4; doi:10.1038/am.2012.6; published online 18 January 2012

Keywords: chiral materials; chiral recognition; smart polymers

\section{INTRODUCTION}

Chiral recognition and enantioselective interaction among molecules are fundamentally important in natural biological processes and in a wide variety of chemical engineering and industrial applications. ${ }^{1,2}$ In chemistry, these processes are usually studied by optical or spectroscopic methods. However, it is more desirable to transform the chiral signals of molecules into changes in the macroscopic properties of a material, which will offer extra benefits for practical applications, including chiral separation, chiral catalysis and functional devices. ${ }^{3}$ In this respect, chiral phenomena in nature are enlightening. For example, chiral amino acids are the basic building blocks of proteins and enzymes, in which chirality not only results in the secondary $\alpha$ helix or $\beta$-sheet structures of proteins, but also determines the higherorder conformations of proteins, as well as their functions and activities. DNA and RNA are composed of 'right-handed' sugars that form helix structures, which have important roles in both gene expression and metabolic pathways. ${ }^{4}$ Chiral biomolecules then assemble to form cells and tissues, which further give rise to the asymmetric shapes or properties of natural biomaterials, for example, the specific rotation directions of shells, conches, snails ${ }^{5}$ and some flowers; ${ }^{6}$ the different tastes or smells of a pair of enantiomers; ${ }^{7}$ and the selective behaviors of cells on enantiomorphous surfaces. ${ }^{8}$
In synthetic systems, although various chiral materials with special properties, for example, chiral liquid crystals (LCs), ${ }^{9}$ chiral metamaterials, ${ }^{10}$ chiral catalysts, ${ }^{11}$ chiral bio-sensors ${ }^{12}$ and chiral separation materials, ${ }^{13}$ have been developed; the study of such materials is still challenging for chemists and materials scientists. Among these materials, smart polymers are especially of interest owing to the fine control of their functions by external stimuli (such as temperature, solvent, $\mathrm{pH}$, ionic strength, light and electric fields) and the easy tunability of these polymers through altering their chemical compositions. ${ }^{14,15}$ These materials provide an ideal platform for the integration of chiral acceptor units through copolymerization with other units; this integration allows the transfer of chiral signals from either guest chiral molecules or the polymers themselves, to the macroscopic properties of the material.

On one hand, the helical structure and the assembly of polymer chains that contain stimuli-responsive units can be mediated by external stimuli, ${ }^{16}$ which directly influence the chiral conformation of polymer chains at the macromolecular level, resulting in obvious changes in the macroscopic properties of the materials. On the other hand, stereoselective interactions between chiral guest molecules and smart polymers could also induce the formation of helical structures of polymer chains or trigger a conformational change in the helical

\footnotetext{
${ }^{1}$ State Key Laboratory of Advanced Technology for Materials Synthesis and Processing, Wuhan University of Technology, Wuhan, China; ${ }^{2}$ Department of Chemistry, Wuhan University of Technology, Wuhan, China and ${ }^{3}$ Physikalisches Institut, Münster, Germany

Correspondence: Professor T Sun, State Key Laboratory of Advanced Technology for Materials Synthesis and Processing, Wuhan University of Technology, 122 Luoshi Road, Wuhan 430070, China.

E-mail: suntaolei@iccas.ac.cn

Received 5 October 2011; revised 3 November 2011; accepted 8 November 2011
} 
structures. Polymer helices further self-assemble into single-handed helical supramolecular assemblies and exhibit the corresponding macroscopic properties, ${ }^{17}$ such as morphology, a liquid-crystalline phase, conductivity, swelling of the hydrogel and wettability. Moreover, investigations of how the chirality of polymer films influences the macroscopic behavior of biological or biochemical entities such as cells, DNA and proteins has become an important and challenging topic, which has led to the appearance of a new research field: chiral biointerface materials. ${ }^{18,19}$

In this review, we will summarize recent progress in these areas. We will focus on the chirality-induced transformation of the macroscopic properties of smart polymer materials and their stepwise chiral transformation processes from the single-molecule level to the macroscopic level. The outline of this review is illustrated in Figure 1.

\section{EXTERNAL STIMULI-MEDIATED TRANSFORMATION OF CHIRAL CONFORMATIONS IN SMART POLYMERS}

Smart polymers can respond to external stimuli reversibly and thus, can be used to trigger special changes in the structures and functions of materials. The introduction of chirality into the backbone or side chains of smart responsive polymers helps to realize reversible chiral modulation of the helix conformations by external stimuli, for example, temperature, solvent, $\mathrm{pH}$ and light irradiation, which provide a satisfactory platform for mimicking chiral behaviors in nature. ${ }^{20}$

\section{Thermo-mediated transformation}

Temperature is one of most common stimuli in nature; it provides a convenient method to mediate the chiral conformation of polymers. In an early study by Tang et al., ${ }^{21}$ the relationship between temperature and chiroptical activity of LCs was evaluated systematically. They found that an LC phase could be observed with the naked eye using crossed polarizers when the temperature was near the nematic temperature of the material. Subsequently, Sakai et al. ${ }^{22}$ reported host-guest complex formation between poly (4'-ethylbenzo-15-crown-5) and L-phenylglycine, which triggered the formation of a one-handed helical structure with an obvious Cotton effect in circular dichroism (CD). Reversible on-off switching of induced CD was mediated by alternating thermo treatments between -30 and $30^{\circ} \mathrm{C}$, which could be repeated for at least five cycles. Similarly, a left-handed helix of poly(phenylacetylene) at lower temperatures could reversibly transform into a right-handed helix at higher temperatures. ${ }^{23} \mathrm{Kim}$ et al. ${ }^{24}$ later described a reversible extension-contraction motion of polymer backbones in response to temperature changes, which resulted in fluorescent switching between enhancement and complete quenching.

Recently, a double-responsive chiral-conjugated system was developed that consisted of a helical polysaccharide (Cur-oeg) chain and a conductive polythiophene (PT1) chain. ${ }^{25}$ The effective conjugation length of PT1 in the complex was modulated by temperature, which led to continuous shifts in the fluorescence and CD spectra during temperature control. Thermo-induced color changes in absorption and fluorescence could be observed clearly with the naked eye, as shown in Figure 2, and showed good reversibility for several thermal cycles. Except for thermal responsiveness, this conjugated film also exhibited color changes when exposed to alternate treatments with water vapor and methanol vapor. The authors presumed that the compact helical conformation of the complex was partially dissociated by the water vapor and then recovered after exposure to methanol vapor. In this exquisite work, thermo- or solvent-mediated chiral helix transitions in the conjugated polymer were expressed as macroscopic color changes in the materials.

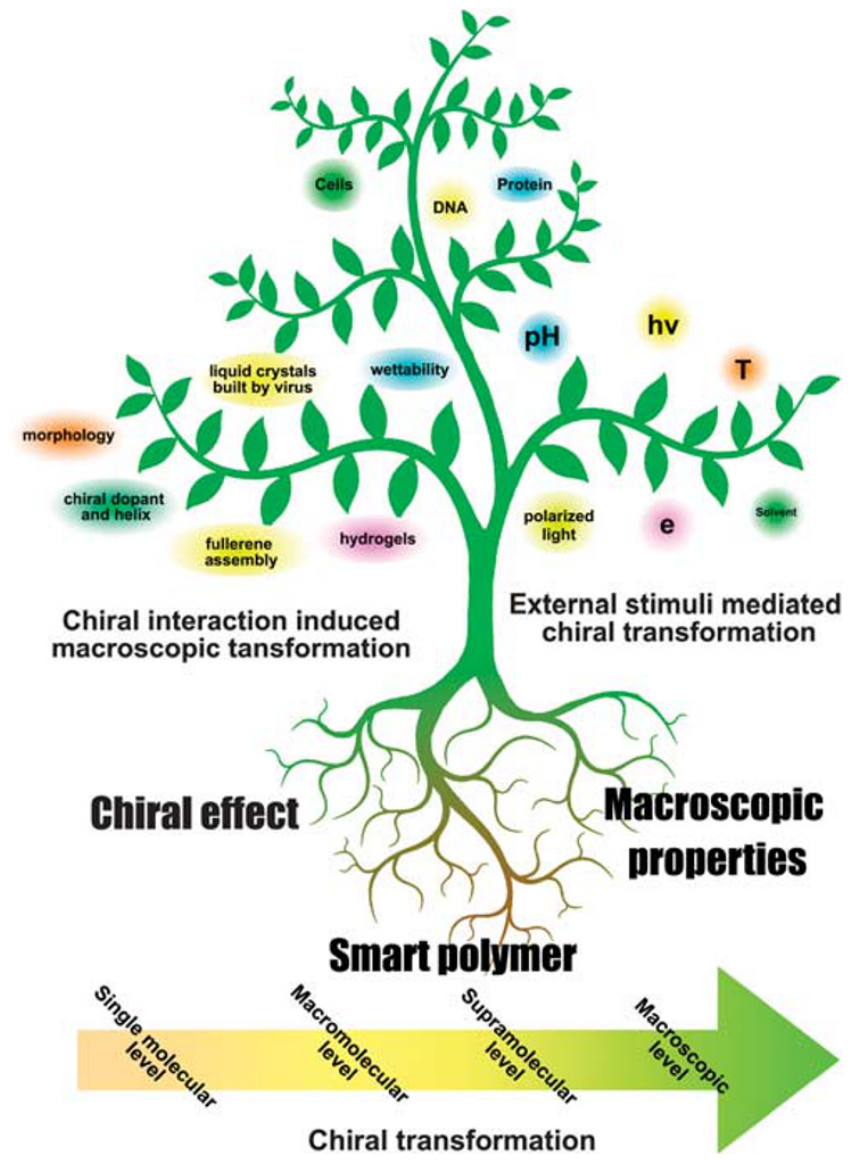

Figure 1 Graphical outline of chirality-responsive polymers that can transform chiral signals from single-molecule chirality into the macroscopic properties of materials.

Thermo-driven molecular motors are another interesting topic. A typical study was reported by Tang et al. ${ }^{26}$ who designed a cylindrical nanoshutter with the capability of transforming lowinput thermal energy or solvent polarity into mechanical output. The key component of this machine was a single screw-sense helical poly(guanidine) with anthracene units. Alternate energy inputs by thermal treatment and solvent polarity actuated the anthracene units in the helix to generate a large-amplitude synchronous waggle. Another interesting molecular machine was developed by Percec et al., ${ }^{27}$ in which self-organized dendronized helical polymers, working as the nanomechanical actuators, underwent a reversible phase transition. This molecular machine, in particular, could exhibit motions at macroscopic length scales in response to temperature changes.

\section{Solvent-mediated transformation}

In addition to temperature, the solvent is another crucial factor that influences the helical structures of polymers. Static diastereomeric leftand right-handed helical poly(isocyanides) have been prepared through control of the polymerization solvent and temperature. ${ }^{28}$ The resultant polymers with opposite helix sense further formed a lyotropic cholesteric LC with opposite twist senses. In their subsequent work, the authors separated single-handed helices from racemic helices through facile treatment with acetone. ${ }^{29}$

In 2006, Sakurai et al. ${ }^{30}$ reported a solvent-mediated helix transition. The helical structure of poly(phenylacetylene) underwent a 


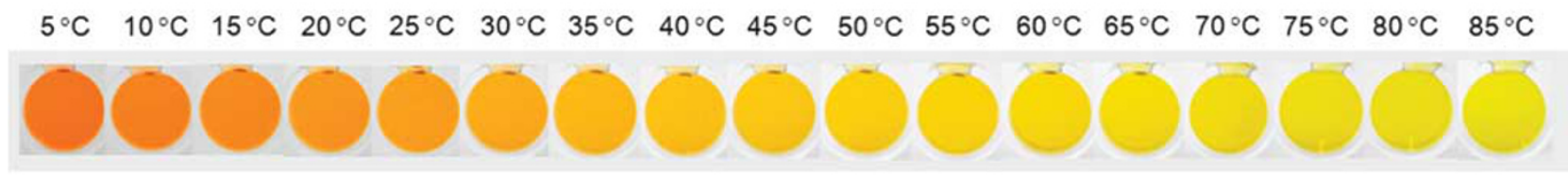

Fluorescence images

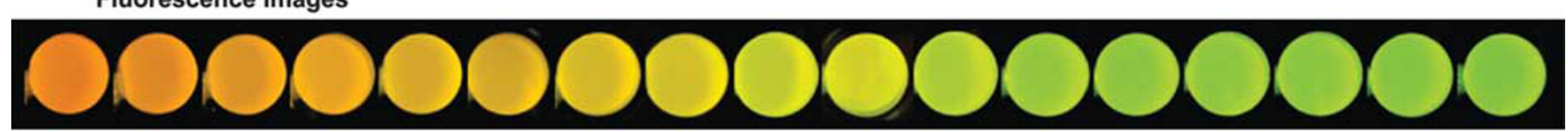

b

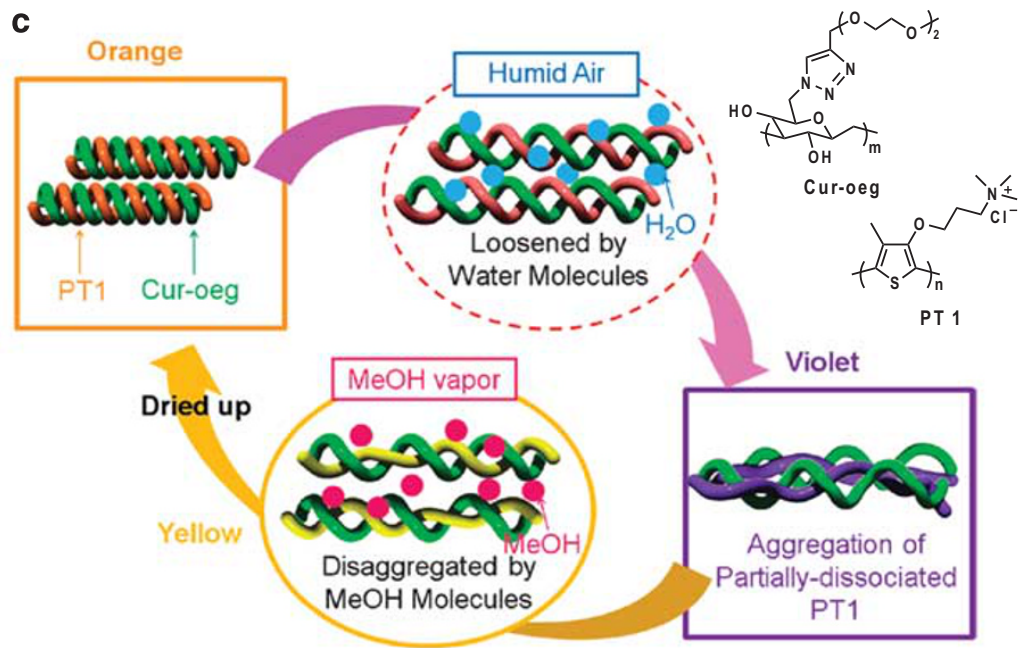

Figure 2 (a) Photographs of polythiophene/polysaccharide (PT1/Cur-oeg) complex solution at different temperatures, from 5 to $85^{\circ} \mathrm{C}$. (b) Photographs of the PT1/Cur-oeg film treated by humid air and methanol vapor and then dried in vacuo. (c) Schematic representation of a solvent-vapor-induced rearrangement of the helical structures of the complex. Adapted from Shiraki et al. ${ }^{25}$ (2010 ACS).

reversible transition from a left-handed helix in benzene to an opposite right-handed helix in tetrahydrofuran and chloroform. The formation of two-dimensional helical bundles with controlled helicity was further induced by the evaporation of solvent. Similar studies have also been independently reported by Zhao et al. ${ }^{31}$ and Yang et al. ${ }^{32}$

\section{pH-mediated transformation}

The $\mathrm{pH}$ value in aqueous media can easily be adjusted, which provides a convenient method for mediating the chirality of materials. In early studies reported by Sanji et al., ${ }^{33}$ a change in the $\mathrm{pH}$ of a solution induced a conformational transition in the host molecule amylase, which further determined the complexation or decomplexation of a guest molecule and resulted in the expression or loss of optical activity. Moreover, $\mathrm{pH}$-responsive hydrogels were found to hierarchically selfassemble into various chiral structures, including helical nanotubes, helical nanotubes with strings of beads, coiled superhelices and dendritic pine- or feather-like nanostructures. These $\mathrm{pH}$-mediated chiral nanostructures could clearly be observed by atomic force microscopy (AFM) ${ }^{34}$ Recently, $\mathrm{pH}$-responsive carboxylic $\beta$-1,3-glucan polysaccharide was used to selectively complex single-walled carbon nanotubes and PT1s in aqueous solution. ${ }^{35}$

More interestingly, natural polypeptides are also $\mathrm{pH}$-responsive systems with helical structures. In 2010, Cho et al. ${ }^{36}$ reported the synthesis of a basic oligopeptide, $\mathrm{Api}_{8}$, composed of eight non-natural achiral amino acids. Compared with natural polypeptides, Api exhibited the opposite $\mathrm{pH}$-responsive behavior and adopted a helical conformation in acid media, which was destroyed in a $\mathrm{pH}$ range of 7-10. In acidic media, hydrogen bonding (H-bonding) interactions between the proximal amide NH protons in the peptide backbone are the critical driving force for the formation of helical peptide structures. However, this H-bonding network would be destroyed in an alkaline medium because non-protonated piperidine groups interact with proximal amide protons, which results in a reversible helix-tocoil transition.

\section{Photo-mediated transformation}

Photo-irradiation is a precise and efficient method for the modulation of the chiral helical structures of polymers. In general, UV irradiation or circularly polarized light (CPL) is introduced as a light stimulus to mediate and enhance the chiral effects of materials. In the exquisite study presented by Pijper et al. ${ }^{37}$ in 2008, UV light was utilized as a stimulus to mediate the chirality of a helix. The authors attached a chiroptical switching molecule at the end of a poly ( $n$-hexyl isocyanate) chain that worked as a light-driven motor. The induction of the chiroptical molecule triggered the conformational transition between $P$-helix and $M$-helix structures through alternate irradiation with UV and visible light. The innovative aspect of their work was that the chiral information was successfully transferred from the macromolecular level of a helical structure to the supramolecular level of a lyotropic cholesteric LC phase. The magnitudes and signs of the helical pitch of the LC phase were accurately controlled using UV $(\lambda=365 \mathrm{~nm})$ or visible light $(\lambda>480 \mathrm{~nm})$; these transition processes 
were clearly observed through an optical microscope equipped with crossed polarizers, as shown in Figure 3. In this work, not only was a photo-mediated chiral transition realized, but chiral information was also expressed at the macroscopic level, which demonstrates a significant advantage of photo-mediated chiral materials and their fascinating application potential.

Similar photo-mediated chiral polymers have been reported by several other groups. ${ }^{38,39}$ In these studies, rapid and reversible photoand thermo-isomerization reactions of azobenzene moieties provided a versatile photo-switchable system and further induced conformational transitions of the polymer backbone. Related studies have been summarized in a review by Choi et al. ${ }^{40}$ in 2007.

The achiral azobenzene homopolymer could not form a helical structure owing to the lack of a chiral auxiliary. However, this situation would be completely different if these homopolymers were irradiated by CPL. In 2007, Zheng et al. ${ }^{41}$ reported an example in which left-CPL irradiation induced a left helical structure in an azopolymer that could be reversed into a right helix under rightCPL irradiation. Tejedor et al. ${ }^{42}$ confirmed this phenomenon and attributed this effect to the chiral arrangement of azobenzene units. The helical direction of the polymer chain was determined by the stereo-regular packing of bisazobenzene chromophores. ${ }^{42,43}$ Owing to the asymmetric photo-isomerization induced by CPL, chirality was transferred from the CPL to the azopolymer through the helical conformation, which further resulted in the macroscopic chiral nematic phase.

In the previously discussed examples, CPL could be used to transfer chirality to an achiral azopolymer chain. However, Satrigo et al. ${ }^{44}$ presented an elegant study in 2006, in which left- or right-CPL was emitted by conjugated poly( $p$-phenylenevinylene), with opposite helical structures in a solid film. The conjugated polymer film was annealed in the presence of chloroform vapor, and the polymer chains gradually self-assembled into a thermodynamically stable $M$-chiral organization and emitted right-CPL. In contrast, an interesting phenomenon was observed when 1,2-dichloroethane was used as the vaporizing solvent: the polymer chains self-assembled into a $P$-chiral organization and emitted predominantly left-CPL. This work thus indicates that the solvent has an important role in the spin-casting process, influencing the structural and electronic properties of the polymer film. However, a single conjugated polymer can emit either right- or left-CPL, depending on the distinct helical conformation of the polymer, and thus, chiral information is transferred from the polymer chains to the macroscopic CPL signal. This result represents a significant discovery for the design of chiral optical materials.

\section{Electric field-mediated transformation}

As an accurate and sensitive modulating method, the mediation of chiral polymers with electric fields is attracting, increasing interest with respect to its potential applications in remote functional devices. In 2008, Song et al. ${ }^{45,46}$ synthesized a helical poly(acetylene) with L-alanine and electrochromic viologens in the side chains. A new negative $\mathrm{CD}$ band at a long wavelength was observed upon reduction of the cationic viologens to radical cations. The intensity of the CD signal switched between 0 and $-1.0 \times 10^{4}$ degree per $\mathrm{cm}^{2} \mathrm{dmol}^{-1}$ during the redox cycle, which could be repeated at least five times. Recently, Ohta et al. ${ }^{47}$ reported on redox-responsive helices with highly condensed $\pi$-clouds. Octameric $o$-phenylene $\mathrm{OP}_{8} \mathrm{NO}_{2}$ helices underwent a rapid helical inversion in solution and formed crystals of both left- and right-handed helices. However, this simple inversion process was prohibited by one-electron oxidation owing to the formation of $\mathrm{OP}_{8} \mathrm{NO}_{2}^{+}$helices with a compact conformation. The helicity was locked into one conformation and exhibited long-lasting chiroptical memory. This interesting study not only provides a novel strategy for the synthesis of one-handed polymers, but also explains from a new perspective why only one-handed helices are present in nature.

\section{CHIRAL INTERACTION-INDUCED MACROSCOPIC PROPERTY TRANSFORMATION}

Enantioselective interactions between chiral molecules and chiral or achiral polymers could induce visible changes in the macroscopic properties of materials, including morphology, liquid-crystalline phase formation, conductivity, swelling of hydrogels and wettability switching. In-depth studies on the chirality transformation processes triggered by chiral interactions will lead to attractive applications in various fields, including chiral biosensors, electronic devices and novel LC materials.

\section{Chiral interaction-induced morphological changes in smart polymers}

Morphology is one of the most typical macroscopic properties of materials. However, only a few chiral helical polymers have been observed to possess a clear helical morphology; most helical polymers lack a distinct morphology owing to the presence of atactic helical arrays at the macromolecular level.

In 2006, Sakurai et al. ${ }^{48}$ reported an ordered two-dimensional crystal with a controlled helical conformation on a graphite surface; the helical structure of the polymer chain was observed with molecular resolution using AFM. The authors subsequently proposed a triplestranded helix model to explain the poly(methyl methacrylate stereocomplex; their model was supported by direct evidence from AFM phase images. ${ }^{49}$ Cheuk et al. ${ }^{50}$ have reported similar results, in which amphiphilic chains of a chiral polymer self-assembled into a variety of organizational morphologies, including twisting cables, spiral ribbons, spherical vesicles and helical nanotubes. Moreover, Goh et al..$^{51}$ investigated the relationship between the concentration of a chiral dopant and the helical pitches of a nematic LC induced by the dopant. The authors used high-resolution scanning electron microscope images to observe a bundle of fibrils in the nematic LC that contained a low concentration of chiral dopant with a helical pitch of $850 \mathrm{~nm}$, whereas single-fibril morphology was observed in the LC that contained a high concentration of dopant with a helical pitch of $270 \mathrm{~nm}$.

\section{Chiral dopant-induced helical morphology and liquid-crystalline phase}

Chiral polyaniline with a helical conformation can be prepared in the presence of a chiral dopant (for example, camphorsulfonic acid). In an early study by Huang et al., ${ }^{52}$ a chiral polyaniline thin film was developed as an enantioselective chiral sensor for amino acids, and phenylalanine enantiomers were discriminated by the macroscopic color changes in the polymer film. In 2007, Yan et al..$^{53}$ prepared an enantiomerically pure helical polyaniline with sub-micrometer tubes and nanostructures of helical nanofibers. Right- and left-handed helical nanofibers were formed using D-camphorsulfonic acid and Lcamphorsulfonic acid as dopants, respectively. Helical nanofibers further self-assembled into helical bundles, which were clearly observed in scanning electron microscope images.

Meanwhile, Nagai et al. ${ }^{54}$ also reported a typical stepwise chiral amplification process induced by a chiral dopant. Taking advantage of the clear changes in the CD spectra and the macroscopic cholesteric twist in the LC state, this hierarchically chiral amplification system could be used to detect small amounts of chiral molecules. A similar 
a
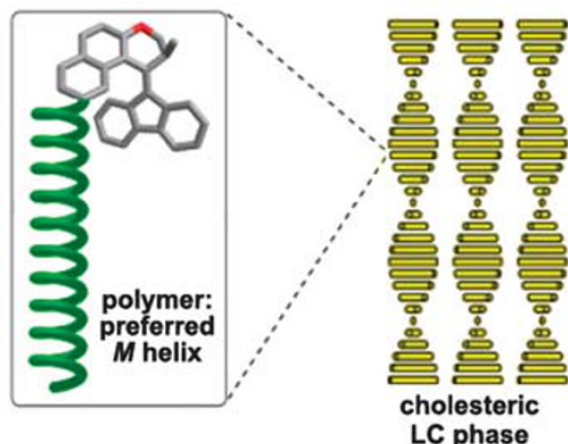

b

(2'S)-(P)-3-PHIC

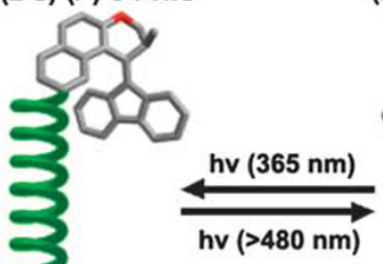

(2'S)-(M)-3-PHIC

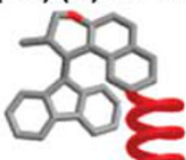

\section{$M$ helix}

d
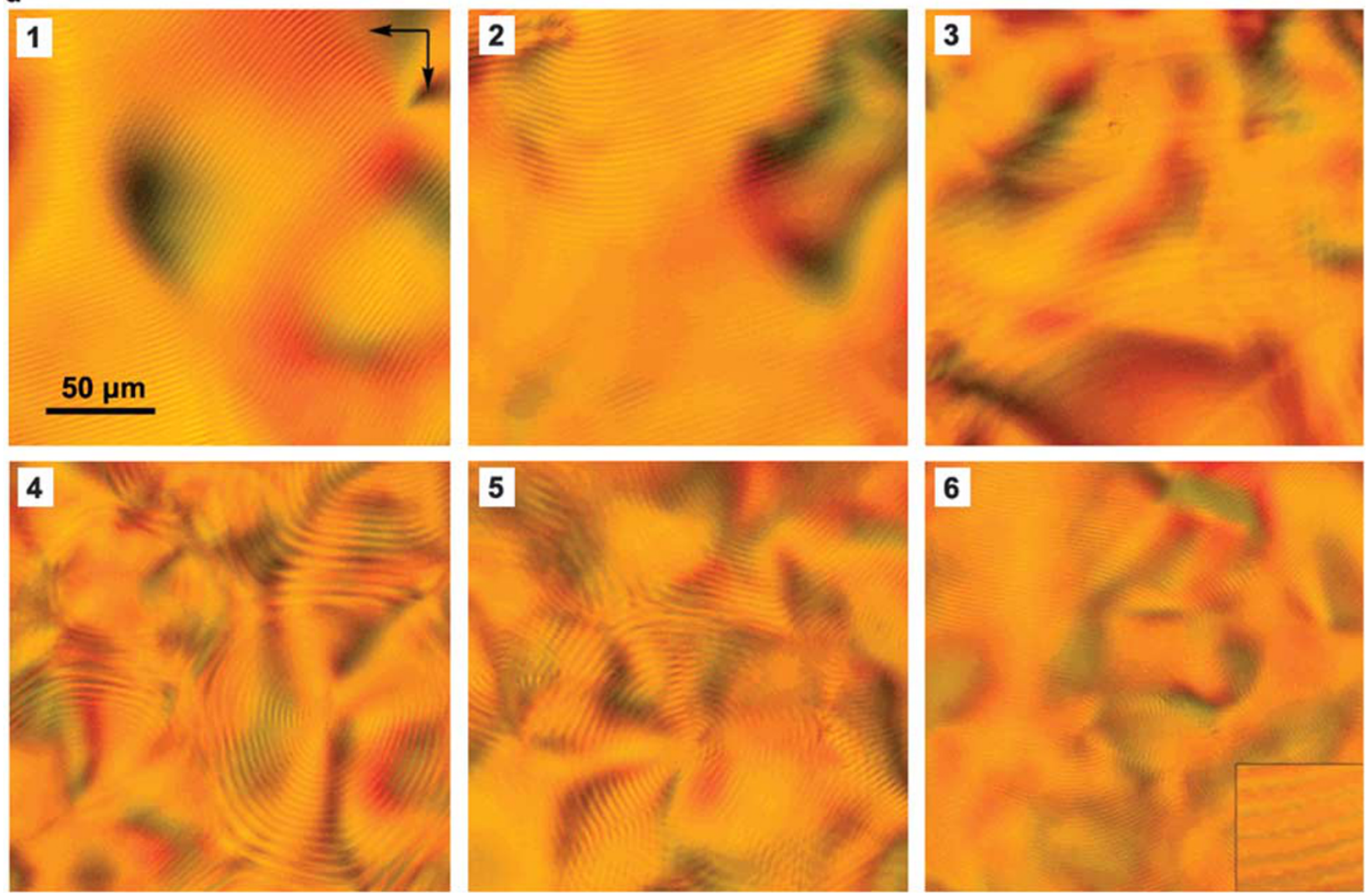

Figure 3 Photo-mediated hierarchical transitions from single-molecule chirality to a macroscopic cholesteric LC phase. (a) Schematic representation of the transition from polyisocyanate with an $M$-helix structure to a cholesteric LC assembly. (b) Schematic representation of photo-mediated conformational transition of the poly(n-hexyl isocyanate) (PHIC) backbone, triggered by the chiroptical molecule appended at the terminus of the polymer chain. (c) Photomediated supramolecular helical pitch of the cholesteric LC phase with different magnitudes and signs. (d) Optical micrographs of a thin film of $\left(2{ }^{\prime} S\right)-(P)$ PHIC in toluene before (1) and after UV irradiation with different exposure times: (2) $25 \mathrm{~min}$, (3) $45 \mathrm{~min}$, (4) $90 \mathrm{~min}$ (5) and 150 min. (6) The irradiated sample left in the dark overnight, which exhibits the transition process from the $P$-helical LC phase to the $M$-helical LC phase, as illustrated in (c). Adapted from Pijper et al. ${ }^{37}$ (2008 ACS). 
supramolecular sensor was designed to recognize various oligosaccharides. ${ }^{55}$ Selective complexation between the sensor and a specific oligosaccharide occurred through noncovalent sugar-aromatic interactions, which destroyed the original homoduplex helix of the sensor and generated a new heteroduplex helix for the complex.

Furthermore, an enantiomer-selective and helix-sense-selective living block copolymerization of isocyanide enantiomers was realized by Wu et al. ${ }^{56}$ In the first stage, right $(P)$ - and left $(M)$-handed helical poly(isocyanides) were prepared by a living polymerization of enantiomerically pure monomer L-1. ${ }^{57}$ In the second stage, living block copolymerization of L-1 or D-1 was initiated by the as-prepared rigid $M$-poly-L-1 or P-poly-L-1 and proceeded in a highly enantiomerselective manner. The $M$-poly-L-1 preferentially copolymerized with L-1 over D-1 by a factor of 6.4-7.7; in contrast, the P-poly-L-1 preferentially copolymerized with D-1 over L-1 by a factor of 4.0. The resultant copolymers adopted almost perfect one-handed helical structures that were determined by the helical chirality of the asprepared poly(isocyanides) used as macroinitiators, as shown in highresolution AFM images. Owing to their rigid rod-like features and narrow molecular-weight distributions, these helical polymers exhibited lyotropic smectic liquid-crystalline phases. Chirality-induced chiroptical property transformations from the poly(isocyanide) macroinitiators to the resultant copolymers were clearly observed in the polarized optical micrographs, as shown in Figures $4 \mathrm{a}-\mathrm{f}$.

\section{Chiral fullerene supramolecular assemblies constructed by helical polymers}

Chiral fullerene supramolecular assembly can be constructed through non-covalent or covalent complexation between a chiral helical polymer and a fullerene. Because it combines a specific supramolecular helical structure with the unique characteristics of a fullerene, the fullerene supramolecular assembly exhibits many specific chemical and physical properties and will find broad applications in chemical sensing, electro-optical devices, enantioselective absorbents and catalysis.

Yashima and coworkers ${ }^{58}$ have published a series of studies on the construction of chiral fullerene supramolecular assemblies through non-covalent complexation. In 2004, they reported that only specific structures and geometries of chiral $\mathrm{C}_{60}$-bisadducts could induce the formation of helical supramolecular assemblies. Subsequently, syndiotactic poly(methyl methacrylate) was used to encapsulate various fullerenes, such as $\mathrm{C}_{60}, \mathrm{C}_{70}$ and $\mathrm{C}_{84}$, within its helical cavity to form a peapod-like crystalline complex; a homogenous film was observed using AFM and high-resolution transmission electron microscope. ${ }^{59}$ Interestingly, the $\mathrm{C}_{60}$ encapsulation process was controlled by chiral phenylethanol, as shown in Figure 5a. The R-enantiomer of phenylethanol induced a right-handed helix, whereas the $S$-enantiomer induced the opposite left-handed helix. The chirality-induced helicity was maintained even after the chiral inducer was removed; the helix therefore exhibited unique 'memorized' characteristics. A similar 'memory' effect was also observed in the complexation of achiral organic dyes by poly(phenylacetylene) helical polymers. ${ }^{60}$ Supramolecular helical J-aggregates of cyanine dyes were formed and exhibited chiroptical activity that remained after the template helix was removed. These studies provide a novel strategy for the construction of chiral helical aggregates with the help of helical polymer templates. ${ }^{61}$ This strategy is especially suitable for achiral molecules or bulky fullerenes and will be advantageous in the development of chiral functional devices.

Yashima et al. ${ }^{62,63}$ have also utilized covalent complexation to construct chiral fullerene supramolecular assemblies in which achiral fullerenes were copolymerized with chiral phenylacetylene components to form stereoregular polymers with fullerene pendants. The prepared copolymers adopted one-handed helical structures, and the fullerene groups were arranged in helical arrays with their screw sense along the polymer backbone. In these studies, chiral phenylacetylene components contributed to the helical structures of supramolecular assemblies. However, achiral phenylacetylene-bearing fullerenes and crown-ether pendants could also form helical arrays upon chiral complexation with L-alanine, as shown in the Figure $5 b^{64}$ The chiral interaction between the L-alanine and the crown-ether pendant ${ }^{65}$ was transmitted to the macromolecular helical structures, which resulted in a helical array of achiral fullerenes along the polymer backbone.

Moreover, many other helical supramolecular assemblies have been reported by several groups. For example, Witte et al. ${ }^{66}$ have synthesized a porphyrin-based helical assembly in which bulky porphyrins are arranged in a left-handed helical array along the polymer backbone. Chiral cyclodextrin residues were also appended to the poly(phenylacetylene) chains and formed a series of one-handed helical conformations. These assemblies exhibited multi-responsiveness toward the temperature and solvent and were capable of complexing chiral or achiral molecules. Such complexation was accompanied by obvious color changes in response to the conformational changes of the helices. ${ }^{67}$

\section{Chirality-responsive polymer hydrogels}

Chirality-responsive polymer hydrogels have emerged as a research field in recent years. ${ }^{68}$ Compared with traditional hydrogel systems, chiral hydrogels exhibit particular helical nanostructures and chiralityresponsive behavior, which can be adjusted by the enantiomeric ratio and stereostructures of gelator units, as well as the chiral guest molecules.

In 2003, Goto et al. ${ }^{69}$ reported the first example of chiralityresponsive polymer hydrogels. The swelling ratio of a hydrogel in dimethyl sulfoxide was dramatically improved by the addition of chiral amines; this improvement was attributed to the formation of one-handed helices through chiral acid-base reactions. Subsequently, Smith et al. ${ }^{70,71}$ developed a chiral hydrogel through the self-assembly of diaminododecane with dendritic peptides that contained three groups of L- or D-lysine building units. By adjusting the molar ratios of L,L,L and D,D,D dendritic peptides to form hydrogels, the authors enabled the molecular chirality of the gelator to determine the extent of fiber formation, the helicity within the fibers and the macroscopic gel strength. In 2005, Morino et al. ${ }^{72}$ further found that the formation of a chiral hydrogel was strongly dependent on the enantiomeric purity of the bis(amino-acid) guest molecule. Furthermore, twistedribbon-like chiral aggregates in hydrogels were clearly observed in the scanning electron microscope images. ${ }^{73}$ Irreversible switching between the gel and the sol was easily controlled by adjusting the $\mathrm{pH}$ value.

More interestingly, polypeptides can also be used to construct chiral hydrogels. Luo et al. ${ }^{74}$ recently designed a chiral peptide, d-EAK 16 , that contains all $\mathrm{D}$-amino acids. This peptide self-assembled into a stable $\beta$-sheet structure and further formed well-ordered nanofibers with the assistance of template metal ions, which resulted in a transparent scaffolded hydrogel. Fibrous structures and macroscopic hydrogels were not observed when d-EAK 16 was composed of alternating D- and L-amino acids because of the reduced chirality of the peptides. In addition, d-EAK 16 with all D-amino acids exhibited a four-fold faster rate in the hemostasis experiment than that of the peptide that contained both L- and D-amino acids. This result provides a novel insight into the design of peptide scaffolds for clinical applications. 
a $M-$ poly-L-1 $100(-)$

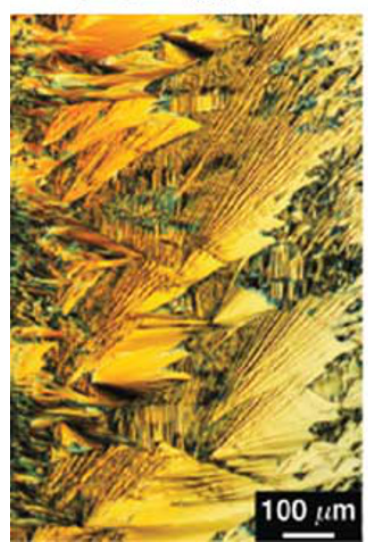

d P-poly-L-1 ${ }_{100}(+)$

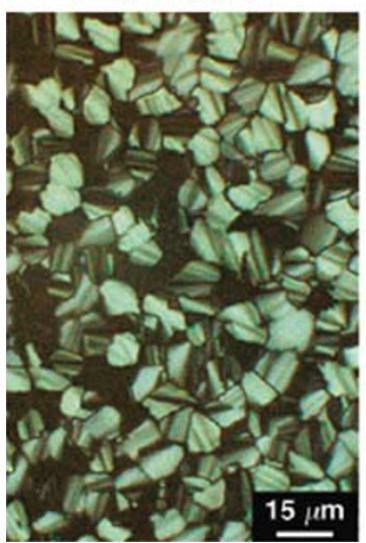

b $M$-poly-L-1 ${ }_{100}(-)-b-L-1$

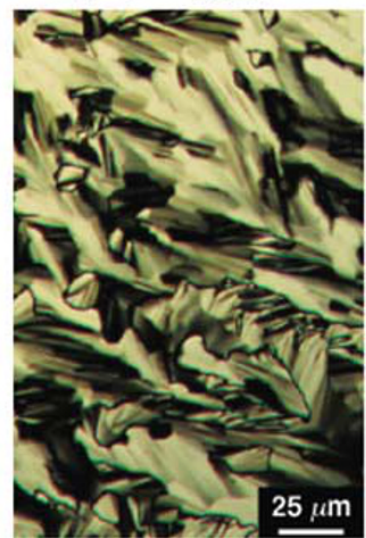

e P-poly-L-1 ${ }_{100}(+)-b-L-1$

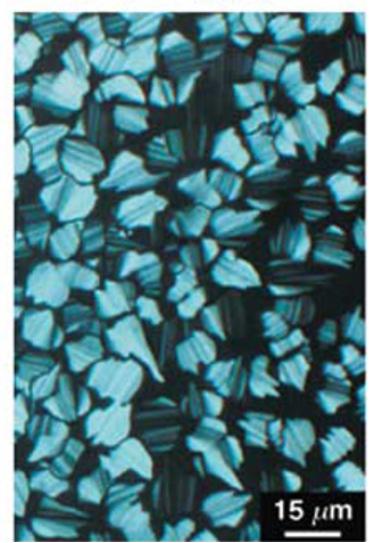

C $M$-poly-L-1 $100(-)-b-D-1$

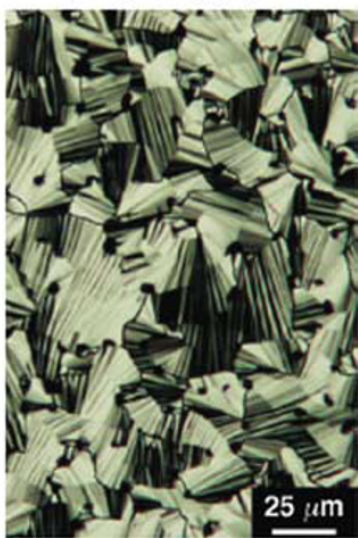

f $P$-poly-L-1 $100(+)-b-D-1$

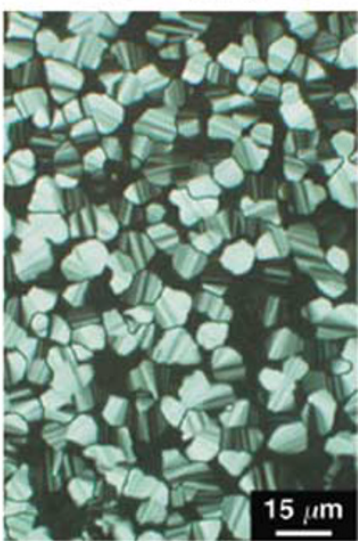

g
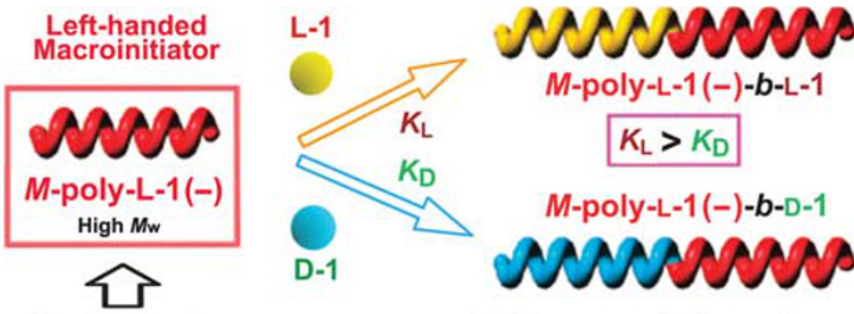

Helix-sense-selective and

Diastereomeric

Helices

ए

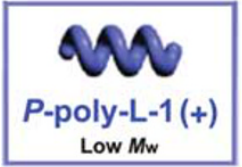

Right-handed Macroinitiator

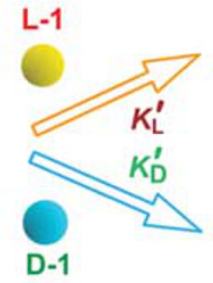

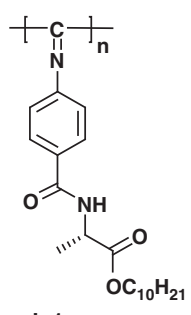

L-1

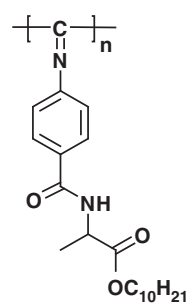

D-1

Figure 4 Polarized optical micrographs of the macroinitiators $M$-poly-L-1 (a) and P-poly-L-1 (b) and the respective block copolymers initiated by the two macroinitiators (c-f). (g) Schematic representation of the enantiomer-selective and helix-sense-selective living block copolymerizations initiated by the macroinitiators with left- and right-handed helical structures. Adapted from Wu et al. ${ }^{56}$ (2009 ACS). 
a

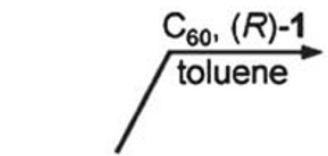

st-PMMA

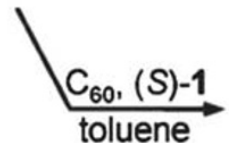

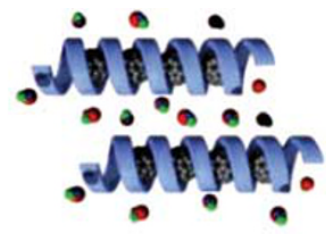

Right-handed helix

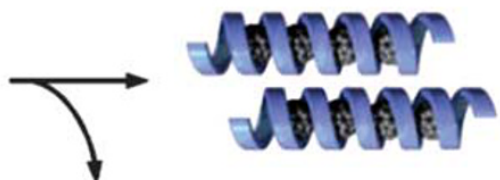

Removal of 1
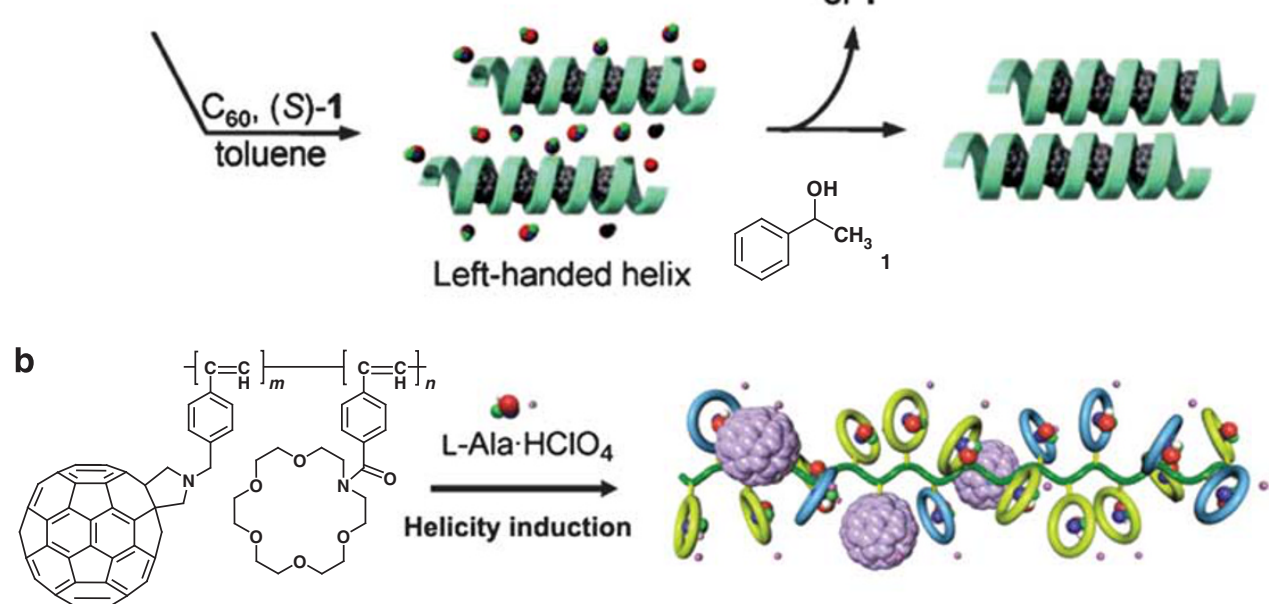

Figure 5 (a) Schematic representation of right- or left-handed helix formation in the $\mathrm{C}_{60}$-encapsulated st-poly(methyl methacrylate) (PMMA) supramolecular assembly induced by $R$ - or $S$-phenylethanol (1). The macromolecular helicity was maintained even after the removal of the chiral dopant. Adapted from Kawauchi et al. ${ }^{59}$ (2008 Wiley-VCH). (b) Schematic representation of the formation of macromolecular helicity in the copolymer with a fullerene pendant induced by complex formation between a crown ether and L-alanine. A chiral crown ether and a fullerene were arranged in helical arrays with the screw-sense along the backbone of the polymer. Adapted from Nishimura et al. ${ }^{64}$ (2004 RSC).

Chirality-triggered wettability switching on smart polymer surfaces Wettability is a fundamental property of a solid surface, and the manipulation of solid-surface wettability has a crucial role in many applications. ${ }^{75}$ Recently, our research group developed a three-component copolymer that contained a $\beta$-Asp-Phe-dipeptide chiral recognition unit, a phenylthiourea-mediating unit and a poly $(\mathrm{N}$ isopropylacrylamide) main chain. ${ }^{76}$ This copolymer was grafted onto a textured silicon substrate composed of nano- and microstructures to form a thin polymer film with a thickness of $20-30 \mathrm{~nm}$. This copolymer film exhibited distinct wettability switching toward the enantiomers of monosaccharides, with lyxose as a typical example; the monosaccharides could be discriminated by the profiles of water droplets on the surface, as shown in Figure 6. Stereoselective complexation between $\beta$-Asp-Phe-dipeptide and L- or D-lyxose was first realized at the molecular level through $\mathrm{H}$-bonding interactions, which further influenced the movements of polymer chains and induced two distinct states of the resultant polymer film: a shrinking state in response to L-lyxose and a swollen state in response to D-lyxose. Conformational transitions of the polymer film determined the surface wettability, through which chiral interaction signals were transmitted into changes in the macroscopic wettability and induced distinct difference in the profiles of water droplets. We also screened numerous dipeptides and monosaccharide enantiomers and found a clear key-to-lock relationship between dipeptide units and monosaccharides, because different molecular structures of dipeptides showed the largest effect for specific kinds of monosaccharide enantiomers. This result represents for the first time that chirality has been expressed as surface wettability, which provides a novel approach for the modulating the wetting properties of materials that deserves special attention in the design of chiral materials.

\section{Chiral LCs constructed by viruses}

LCs can be constructed from many artificial materials, such as chiral helical polymers, chiral supramolecular assemblies and bent-core materials. However, biological viruses can also be used as building blocks to construct chiral LCs, as was reported by Tomar et al. ${ }^{77}$ in 2007. The authors constructed cholesteric LCs from eight phages. The LC properties of the phages were adjusted by the addition of dopants that interacted with the DNA or protein components of viruses. Periodic non-equivalent interactions between the capsids and DNAs in filamentous phages contributed to the large-length-scale coiling, which resulted in the formation of cholesteric LC phases. This research was subsequently advanced by Barry et al. ${ }^{78}$ who used two rod-like viruses ( $f d$ Y21M and $f d$ wild-type (wt)) to construct LCs. The $f d$ wt virus LC exhibited a left-handed cholesteric helix, whereas the $f d$ Y21M virus LC exhibited a right-handed helix and a cholesteric pitch with a magnitude five times greater than that of the $f d$ wt LC, as shown in Figure 7. The macroscopic chirality of the LC system could be precisely controlled by mixing the two viruses. These two viruses were nearly identical; they differed by a single amino acid in the major coat protein pVIII. However, the microscopic difference was successfully amplified to alter the macroscopic helicity of the cholesteric phase. This interesting study provides a novel strategy for controlling the macroscopic structures of chiral assemblies through the modification of viruses with a tunable persistence length and chirality. These phenomena deserve further research to explore and control the transition processes from the microscopic features of biomacromolecules to the macroscopic properties of LCs.

Recently, Chung et al. ${ }^{79}$ advanced this research and explained these phenomena from a new perspective. They also chose a single type of bacterial virus, the M13 phage, as the building block and constructed a 
a

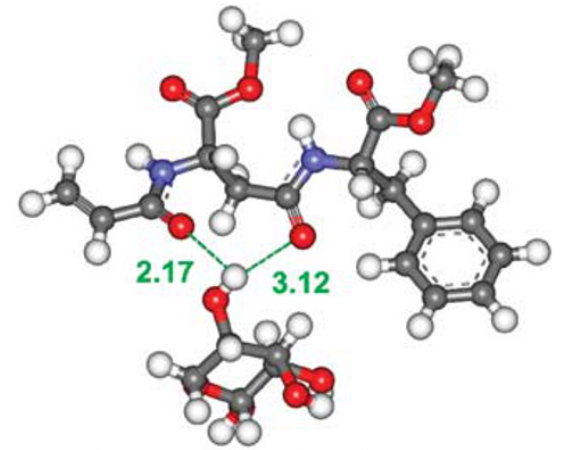

$\beta$-Asp-Phe with L-lyxose

b

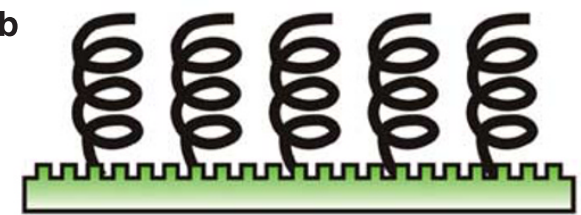

Shrinking state

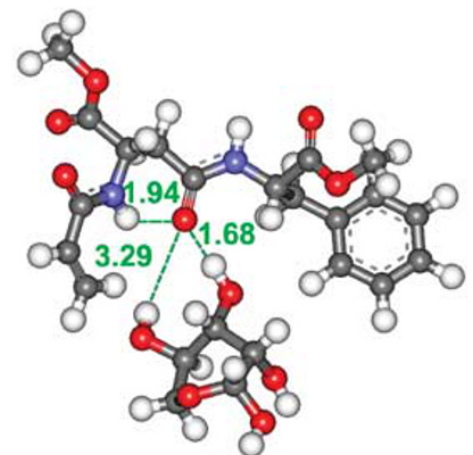

$\beta$-Asp-Phe with D-lyxose

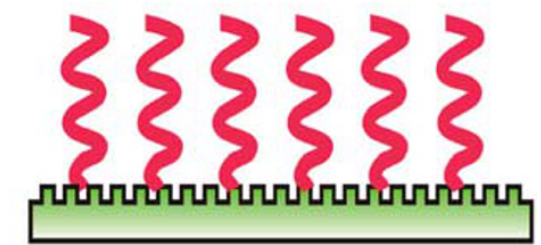

Swollen state
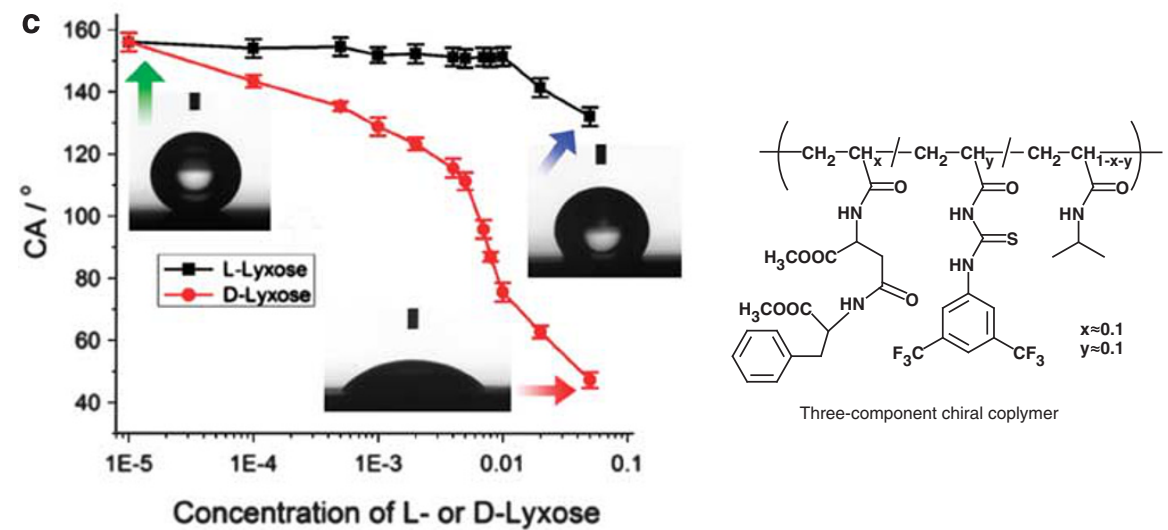

Figure 6 Schematic representation of chirality-induced wettability switching on a chiral smart polymer film containing the $\beta$-Asp-Phe-dipeptide chiral recognition unit. (a) Molecular interaction models of the Asp-Phe-dipeptide with L- or D-lyxose. (b) Shrunken and swollen states of polymer films induced by treatment with L- or D-lyxose solution. (c) Chirality-induced macroscopic wettability discrimination on the polymer film, which was amplified by the structured effect of a textured substrate. Adapted from Qing and $\operatorname{Sun}^{76}$ (2011 Wiley-VCH).
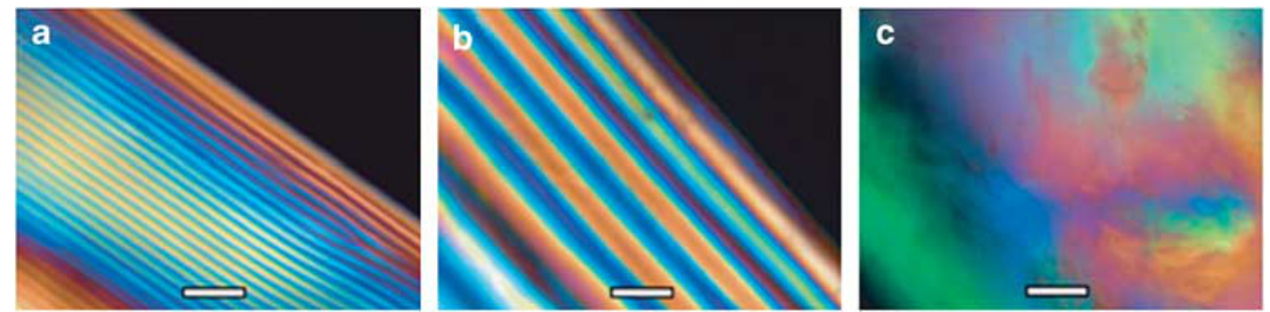

Figure 7 Polarization micrographs of LCs constructed by (a) pure $f d$ wt virus, (b) pure $f d$ Y $21 \mathrm{M}$ virus; (c) a mixture of $20 \% f d$ wt and $80 \% f d$ Y $21 \mathrm{M}$. The scale bars represent $50 \mu \mathrm{m}$. Adapted from Barry et al. ${ }^{78}$ (2009 RSC). 
series of LCs with distinct supramolecular structures by using a selftemplating assembly approach. The phage was assembled into largerarea films by pulling substrates vertically from phage suspensions at precisely controlled speeds. Through precise control of the phage concentration and the pulling speed in the assembly process, chiral LC phases with distinct supramolecular structures, such as nematic orthogonal twists, cholesteric helical ribbons and smectic helicoidal nanofilaments, were formatted. These phases exhibited different optical and photonic properties. More interestingly, cells cultured on these films could recognize the underlying microstructures and grow in multiple directions in a hierarchically organized manner. This work provides a simple fabrication process for the production of complex LCs from a single viral building block. Highly-ordered and controllable structures were obtained on both the sub-micrometer and micrometer scales. This work attempted to address a fundamental problem in nature: how to utilize various environmental factors to control the transformations from helical biomacromolecules to hierarchically structured materials.

\section{CHIRAL BIOINTERFACE MATERIALS}

Stereoselective interactions between chiral materials and biomacromolecules are important in bio-related applications. Although numerous authors have investigated the interfacial interactions of biomaterials with biological systems, few studies have focused on the chirality of biomaterials, especially the chirality-regulated macroscopic behavior of biological or biochemical entities such as cells, DNA and proteins.

In a pioneering work by Addadi et al. ${ }^{8}$ in 1994, Xenopus laevis kidney epithelial A6 cells exhibited different cell adhesion on (011) faces of $(R, R)$ and $(S, S)$ calcium tartrate tetrahydrate crystals. In 2003, the authors continued their research to investigate the binding properties of two antibodies to the surfaces of chiral enantiomers. ${ }^{80}$ Stereoselective recognition and complementary bonding occurred between antibody $48 \mathrm{E}$ and a (L)-leucine-(L)-leucine-(L)-tyrosine surface; however, this interaction was suppressed by the enantiomeric (D)-Leu-(D)-Leu-(D)-Tyr surface. The authors then built a series of molecular models to explain this chiral effect. ${ }^{81} \mathrm{H}$-bonding and hydrophobic interactions, as well as $\pi-\pi$ stacking between aromatic rings, were described clearly in the molecular models to illustrate the details of the stereoselective interactions.

Our research group further introduced chiral monolayer surfaces and chiral polymer brushes into this study, which significantly broadened the scope of the research and opened the new research field of chiral biointerface materials. ${ }^{19}$ We reported in 2007 that two important immune cells-macrophages and neutrophils-exhibited significantly less cell adhesion and activation on an $N$-isobutyryl-Dcysteine (D-NIBC)-modified surface than on an L-NIBC-modified surface. This chiral effect was amplified to a certain extent on the nanostructured substrate. ${ }^{82}$ Subsequently, a single-stranded DNA from calf thymus was observed to have a stronger interaction with the L-NIBC surface than with the corresponding D-surface. ${ }^{83}$ The adsorbed single-stranded DNA inclined to form a relaxed supercoillike structure on the L-surface, whereas it had a folded particle-like morphology on the D-surface. Moreover, double-stranded DNA from the plasmid pcDNA3 was also found to exhibit similar stereoselective behaviors on L(D)-NIBC-modified surfaces. ${ }^{84}$ The L-NIBC surface exhibited a strong interaction with double-stranded DNA and induced a transformation of double-stranded DNA from the supercoiled conformation to the relaxed conformation, as shown in Figures 8a and $\mathrm{b}$. In contrast, the interaction with the D-surface was weaker, and more adsorbed double-stranded DNA maintained its initial super-coiled rod-like conformation. Dynamic and theoretical analyses indicated that this process was dominated by the stereo-specific $\mathrm{H}$ bonding interactions between the chiral moieties of DNAs and the surface. Recently, Li et al. ${ }^{85}$ found that L- and D-glutathione (GSH)modified CdTe quantum dots (QDs) exhibited different cytotoxicity toward cells and induced distinct autophagy in cells. L-GSH-QDs induced more obvious LC3-enhanced green fluorescent protein aggregation in HepG2 cells than did D-GSH-QDs, as shown in Figures 8c and d. After HepG2 cells were cultured on chiral QD surfaces for $24 \mathrm{~h}$, a large number of empty vacuoles were observed in the cytoplasm, as shown in the transmission electron microscope images (Figures $8 \mathrm{e}$ and $\mathrm{f}$ ); however, unlike L620-QDs $\left(\lambda_{\max }=620 \mathrm{~nm}\right)$, the D622-QDs $\left(\lambda_{\max }=622 \mathrm{~nm}\right)$ could not induce sufficient vacuole formation.

Compared with self-assembled monolayers, chiral polymer films are thought to exhibit more obvious chiral effects. In 2010, our group prepared a chiral polyvaline film, based on the enantiomers of valine, and evaluated the behavior of cells on the chiral polymer surfaces. ${ }^{86}$ We found that both COS-7 and bEnd. 3 cells were able to adhere, grow, spread and assemble much better on the L-valine-based polymer film than on the corresponding D-film. Recently, we extended our research to the protein level and evaluated the influence of surface chirality on the adsorption and interaction of proteins. An L-polyvaline polymer film exhibited a stronger affinity for bovine serum albumin than did a D-polyvaline film. ${ }^{87}$ However, this chiral effect was not observed on the self-assembled monolayer surface modified by chiral valine. Thermodynamic analysis indicated that the stereoselective interaction between the chiral polymers and the proteins is a process dominated by stereoselective hydrophobic interactions, not a process dominated by $\mathrm{H}$-bonding. Meanwhile, Yi et al. ${ }^{88}$ reported a similar study, and their results are consistent with the experimental findings of our group. They found that a poly(D-lactide) film exhibited weaker effects on cell attachment, proliferation and growth than did an enantiomeric poly(L-lactide) film. The total protein amount, DNA content and alkaline phosphatase activity of ROS 17/2.8 cells on the poly(D-lactide) film were also lower than those on the poly(L-lactide) film.

These studies have proved that chirality can influence the macroscopic behavior of biomacromolecules. However, such research is still in its early stages. In the future, this technology is expected to find additional applications in protein regulation, tissue engineering, chiral drug release and bio-devices.

\section{CONCLUSIONS AND PERSPECTIVE}

Recent progress in chirality-responsive polymers has attracted broad interest owing to the ability of these polymers to transform chiral signals into changes in macromolecular structures, conformations and the corresponding macroscopic properties of materials. When properly designed, the chirality of polymers can be mediated by external stimuli or chiral guest molecules in an intelligent mode. Taking advantage of the distinct changes in the macroscopic properties of materials, chirality-responsive polymers are having increasingly important roles in a wide range of applications, such as biosensors, chiral optical and electronic devices, a new generation of LC materials, chiral separation membranes, nanomachines, microfluidic devices and bio-chips.

Nevertheless, many challenges remain to be solved in this field. The first challenge is the determination of the mechanism of chiral transformation, especially the dynamic process of chirality transfer and the instantaneous change in conformation of the polymer. We emphasize that the chiral transformation process is more complex than has previously been thought, and more fantastic phenomena 

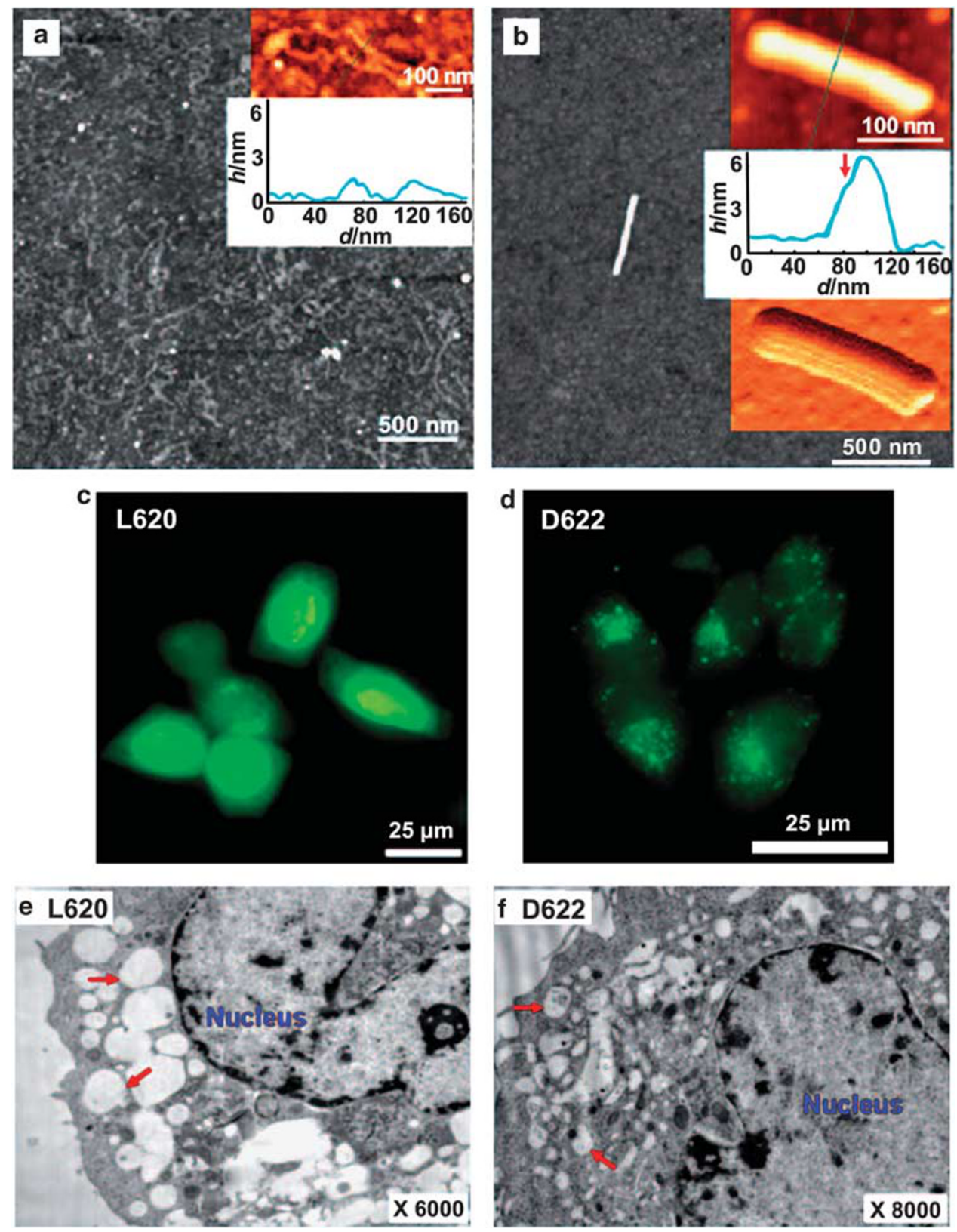

Figure $8(\mathbf{a}, \mathbf{b})$ AFM images for double-stranded pcDNA3 adsorption on a gold surface modified by L-NIBC (a) or D-NIBC (b) monolayers. The inset in (a) is the magnified image of single relaxed-circular DNA on the L-surface; the inset in (b) is the magnified image of a supercoiled DNA rod on the D-surface. Adapted from Gan et al..$^{84}$ (2010 Wiley-VCH). (c, d) Fluorescence microscopy images of LC3-enhanced green fluorescent protein (EGFP) aggregation that was induced in HepG2 cells by treatment with L-GSH-QDs (c) or D-GSH-QDs (d). (e, f) transmission electron microscopy (TEM) images of HepG2 cells cultured on the chiral QD surfaces for $24 \mathrm{~h}$. QD-induced autophagic cell death was evident from numerous large vacuoles within the cytoplasm, as indicated by red arrows. Adapted from Li et al. ${ }^{85}$ (2011 Wiley- $\mathrm{VCH}$ ).

await exploration by scientists. Ohta et al. ${ }^{47}$ have already established a good example in slowing the helical transition and 'freezing' the helical structure in a one-handed phase. The second challenge is that external stimuli could induce reversible transformations in the macroscopic properties of materials. However, for the development of practical nanomachines and nanodevices, the driving force is too low to actuate a nanomachine to operate continuously, because the stimulus-induced macroscopic transformation is still very limited. The response speed is another important factor that needs to be considered in practical applications. To solve these problems, more creative chiral materials should be developed, and close collaborations between chemists and materials scientists are extremely important for this research. The third challenge is the utilization of the chirality of polymer materials to regulate the behavior and functions of biomacromolecules. For example, can we regulate the conformational transitions in peptides through stereoselective H-bonding or hydrophobic interactions? This is a meaningful and significant research topic, and chirality-responsive materials will be the best option for tackling this challenge, because they undergo transformations similar to those of their natural counterparts. 


\section{ACKNOWLEDGEMENTS}

We thank the National Natural Science Foundation of China (51073123) and the Fundamental Research Funds for the Central Universities of China (2010ZX-025) for funding support.

1 Berthod, A. Chiral recognition mechanisms. Anal. Chem. 78, 2093 (2006)

2 Hazen, R. M. \& Sholl, D. S. Chiral selection on inorganic crystalline surfaces. Nat. Mater. 2, 367 (2003).

3 Fasel, R., Parschau, M. \& Ernst, K. H. Amplification of chirality in two-dimensional enantiomorphous lattices. Nature 439, 449 (2006).

4 Berg, J. M., Tymoczko, J. L. \& Stryer, L. Biochemistry (5th Ed.) (W. H. Freeman: New York, USA, 2002).

5 Grande, C. \& Patel, N. H. Nodal signalling is involved in left-right asymmetry in snails. Nature 457, 1007 (2009).

6 Jesson, L. K. \& Barrett, S. C. H. Enantiostyly: solving the puzzle of mirror-image flowers. Nature 417, 707 (2002).

7 Friedman, L. \& Miller, J. G. Odor incongruity and chirality. Science 172, 1044 (1971).

8 Hanein, D., Geiger, B. \& Addadi, L. Differential adhesion of cells to enantiomorphous crystal surfaces. Science 263, 1413 (1994).

9 O'Neill, M. \& Kelly, S. M. Liquid crystals for charge transport, luminescence, and photonics. Adv. Mater. 15, 1135 (2003).

10 Zheludev, N. I. The road ahead for metamaterials. Science 328, 582 (2010).

11 Taylor, M. S. \& Jacobsen, E. N. Asymmetric catalysis by chiral hydrogen-bond donors. Angew. Chem. Int. Ed. 45, 1520 (2006)

$12 \mathrm{Pu}$, L. Fluorescence of organic molecules in chiral recognition. Chem. Rev. 104, 1687 (2004).

13 Maier, N. M., Franco, P. \& Lindner, W. Separation of enantiomers: needs, challenges, perspectives. J. Chromatogr. A. 906, 3 (2001).

14 Lendlein, A. \& Shastri, V. P. Stimuli-sensitive polymers. Adv. Mater. 22, 3344 (2010).

15 Stuart, M. A. C., Huck, W. T. S., Genzer, J., Müller, M., Ober, C., Stamm, M. Sukhorukov, G. B., Szleifer, I., Tsukruk, V. V., Urban, M., Winnik, F., Zauscher, S., Luzinov, I. \& Minko, S. Emerging applications of stimuli-responsive polymer materials. Nat. Mater. 9, 101 (2010).

16 Sun, T. \& Qing, G. Biomimetic smart interface materials for biological applications. Adv. Mater. 23, H57 (2011).

17 Yashima, E., Maeda, K., lida, H., Furusho, Y. \& Nagai, K. Helical polymers: synthesis, structures, and functions. Chem. Rev. 109, 6102 (2009).

18 Nel, A. E., Mädler, L., Velegol, D., Xia, T., Hoek, E. M. V., Somasundaran, P., Klaessig, F., Castranova, V. \& Thompson, M. Understanding biophysicochemical interactions at the nano-bio interface. Nat. Mater. 8, 543 (2009).

19 Zhang, M. X., Qing, G. Y. \& Sun, T. L. Chiral biointerface materials. Chem. Soc. Rev. doi: 10.1039/C1CS15209B (2012).

20 Liu, F. \& Urban, M. W. Recent advances and challenges in designing stimuli-responsive polymers. Prog. Polym. Sci. 35, 3 (2010).

21 Tang, K., Green, M. M., Cheon, K. S., Selinger, J. V. \& Garetz, B. A. Chiral conflict. The effect of temperature on the helical sense of a polymer controlled by the competition between structurally different enantiomers: from dilute solution to the lyotropic liquid crystal state. J. Am. Chem. Soc. 125, 7313 (2003).

22 Sakai, R., Otsuka, I., Satoh, T., Kakuchi, R., Kaga, H. \& Kakuchi, T. Thermoresponsive on-off switching of chiroptical property induced in poly(4'-ethynylbenzo-15-crown-5)/ $\alpha$ amino acid system. Macromolecules 39, 4032 (2006).

23 Miyagawa, T., Furuko, A., Maeda, K., Katagiri, H., Furusho, Y. \& Yashima, E. Dual memory of enantiomeric helices in a polyacetylene induced by a single enantiomer. J. Am. Chem. Soc. 127, 5018 (2005).

$24 \mathrm{Kim}, \mathrm{H}$. J., Lee, E., Park, H. S. \& Lee, M. Dynamic extension-contraction motion in supramolecular springs. J. Am. Chem. Soc. 129, 10994 (2007).

25 Shiraki, T., Dawn, A., Tsuchiya, Y. \& Shinkai, S. Thermo- and solvent-responsive polymer complex created from supramolecular complexation between a helix-forming polysaccharide and a cationic polythiophene. J. Am. Chem. Soc. 132, 13928 (2010).

26 Tang, H. Z., Novak, B. M., He, J. T. \& Polavarapu, P. L. A thermal and solvocontrollable cylindrical nanoshutter based on a single screw-sense helical polyguanidine. Angew. Chem. Int. Ed. 44, 7298 (2005).

27 Percec, V., Rudick, J. G., Peterca, M. \& Heiney, P. A. Nanomechanical function from self-organizable dendronized helical polyphenylacetylenes. J. Am. Chem. Soc. 130, 7503 (2008).

28 Kajitani, T., Okoshi, K., Sakurai, S. I., Kumaki, J. \& Yashima, E. Helix-sense controlled polymerization of a single phenyl isocyanide enantiomer leading to diastereomeric helical polyisocyanides with opposite helix-sense and cholesteric liquid crystals with opposite twist-sense. J. Am. Chem. Soc. 128, 708 (2006)

29 Onouchi, H., Okoshi, K., Kajitani, T., Sakurai, S. I., Nagai, K., Kumaki, J., Onitsuka, K. \& Yashima, E. Two- and three-dimensional smectic ordering of single-handed helical polymers. J. Am. Chem. Soc. 130, 229 (2008).

30 Sakurai, S. I., Okoshi, K., Kumaki, J. \& Yashima, E. Two-dimensional surface chirality control by solvent-induced helicity inversion of a helical polyacetylene on graphite. $J$. Am. Chem. Soc. 128, 5650 (2006).

31 Zhao, H. C., Sanda, F. \& Masuda, T. Transformation of helical sense of poly $(\mathrm{N}$ propargylamides) controlled by competition between structurally different enantiomeric amino acids. Macromolecules 37, 8888 (2004).

32 Yang, C. T., Wang, Y. L. \& Chang, Y. C. Effect of solvents and temperature on the conformation of poly( $\beta$-benzyl-I-aspartate) brushes. Biomacromolecules 11, 1308 (2010).
33 Sanji, T., Kato, N. \& Tanaka, M. Switching of optical activity in oligosilane through pHresponsive chiral wrapping with amylose. Macromolecules 39, 7508 (2006).

34 Duan, P. F., Qin, L., Zhu, X. F. \& Liu, M. H. Hierarchical self-assembly of amphiphilic peptide dendrons: Evolution of diverse chiral nanostructures through hydrogel formation over a wide pH range. Chem. Eur. J. 17, 6389 (2011).

35 Lien le, T. N., Shiraki, T., Dawn, A., Tsuchiya, Y., Tokunaga, D., Tamaru, S., Enomoto, N., Hojo, J. \& Shinkai, S. A pH-responsive carboxylic $\beta$-1,3-glucan polysaccharide for complexation with polymeric guests. Org. Biomol. Chem. 9, 4266 (2011).

36 Cho, J. I., Tanaka, M., Sato, S., Kinbara, K. \& Aida, T. Oligo(4-aminopiperidine-4carboxylic acid): an unusual basic oligopeptide with an acid-induced helical conformation. J. Am. Chem. Soc. 132, 13176 (2010)

37 Pijper, D., Jongejan, M. G. M., Meetsmia, A. \& Feringa, B. L. Light-controlled supramolecular helicity of a liquid crystalline phase using a helical polymer functionalized with a single chiroptical molecular switch. J. Am. Chem. Soc. 130, 4541 (2008).

38 Zhao, H. C., Sanda, F. \& Masuda, T. Stimuli-responsive conjugated polymers. Synthesis and chiroptical properties of polyacetylene carrying L-glutamic acid and Azobenzene in the side chain. Polymer 47, 2596 (2006).

39 Ouchi, Y., Morisaki, Y., Ogoshi, T. \& Chujo, Y. Synthesis of a stimuli-responsive P-chiral polymer with chiral phosphorus atoms and azobenzene moieties in the main chain. Chem. Asian J. 2, 397 (2007).

40 Choi, S. -W., Kawauchi, S., Ha, N. Y. \& Takezoe, H. Photoinduced chirality in azobenzene-containing polymer systems. Phys. Chem. Chem. Phys. 9, 3671 (2007).

41 Zheng, Z., Su, Z., Wang, L., Xua, J., Zhang, Q. \& Yang, J. Photoinduced chirality in achiral liquid crystalline azobenzene polymers containing different polar side groups. Euro. Polym. J. 43, 2738 (2007).

42 Tejedor, R. M., Oriol, L., Serrano, J. L., Ureña, F. P. \& González, J. J. L. Photoinduced chiral nematic organization in an achiral glassy nematic azopolymer. Adv. Funct. Mater. 17, 3486 (2007).

43 Pan, X., Jiang, H., Wang, Y., Lei, Z., Zou, G., Zhang, Q. \& Wang, K. Supramolecular chirality formation of bisazobenzene-substituted polydiacetylene LB films. J. Colloid Interface Sci. 354, 880 (2011).

44 Satrijo, A., Meskers, S. C. J. \& Swager, T. M. Probing a conjugated polymer's transfer of organization-dependent properties from solutions to films. J. Am. Chem. Soc. 128 , 9030 (2006).

45 Deng, J., Zhou, C., Chen, C., Song, N. H. \& Su, Z. X. Synthesis and redox-driven chiroptically switching properties of viologen-containing optically active polymer with main-chain axial chirality. Macromolecules 41, 7805 (2008).

46 Deng, J., Zhou, C. \& Song, N. H. Chiroptically active photonics polymers: synthesis and chiroptically switching properties of helical polyacetylene bearing electrochromic viologens in the side chains. Macromolecules 42, 6865 (2009).

47 Ohta, E., Sato, H., Ando, S., Kosaka, A., Fukushima, T., Hashizume, D., Yamasaki, M., Hasegawa, K., Muraoka, A., Ushiyama, H., Yamashita, K. \& Aida, T. Redox-responsive molecular helices with highly condensed $\pi$-clouds. Nat. Chem. 3, 68 (2011).

48 Sakurai, S. I., Okoshi, K., Kumaki, J. \& Yashima, E. Two-dimensional hierarchical selfassembly of one-handed helical polymers on graphite. Angew. Chem. Int. Ed. 45, 1245 (2006).

49 Kumaki, J., Kawauchi, T., Okoshi, K., Kusanagi, H. \& Yashima, F. Supramolecular helical structure of the stereocomplex composed of complementary isotactic and syndiotactic poly(methyl methacrylate)s as revealed by atomic force microscopy. Angew. Chem. Int. Ed. 46, 5348 (2007).

50 Cheuk, K. K. L., Li, B. S., Lam, J. W. Y., Xie, Y. \& Tang, B. Z. Synthesis, chain helicity, assembling structure, and biological compatibility of poly(phenylacetylene)s containing I-alanine moieties. Macromolecules 41, 5997 (2008).

51 Goh, M., Kyotani, M. \& Akagi, K. Highly twisted helical polyacetylene with morphology free from the bundle of fibrils synthesized in chiral nematic liquid crystal reaction field. J. Am. Chem. Soc. 129, 8519 (2007).

52 Huang, J., Egan, V. M., Guo, H., Yoon, J. Y., Briseno, A. L., Rauda, I. E., Garrell, R. L., Knobler, C. M., Zhou, F. \& Kaner, R. B. Enantioselective discrimination of D- and L-phenylalanine by chiral polyaniline thin films. Adv. Mater. 15, 1158 (2003).

53 Yan, Y., Yu, Z., Huang, Y. W., Yuan, W. X. \& Wei, Z. X. Helical polyaniline nanofibers induced by chiral dopants by a polymerization process. Adv. Mater. 19, 3353 (2007).

54 Nagai, K., Sakajiri, K., Maeda, K., Okoshi, K., Sato, T. \& Yashima, E. Hierarchical amplification of macromolecular helicity in a lyotropic liquid crystalline charged poly(phenylacetylene) by nonracemic dopants in water and its helical structure. Macromolecules 39, 5371 (2006)

55 Goto, H., Furusho, Y. \& Yashima, E. Double helical oligoresorcinols specifically recognize oligosaccharides via heteroduplex formation through noncovalent interactions in water. J. Am. Chem. Soc. 129, 9168 (2007).

56 Wu, Z. Q., Nagai, K., Banno, M., Okoshi, K., Onitsuka, K. \& Yashima, E. Enantiomerselective and helix-sense-selective living block copolymerization of isocyanide enantiomers initiated by single-handed helical poly(phenyl isocyanide)s. J. Am. Chem. Soc. 131,6708 (2009)

57 Onouchi, H., Okoshi, K., Kajitani, T., Sakurai, S. I., Nagai, K., Kumaki, J., Onitsuka, K. \& Yashima, E. Two- and three-dimensional smectic ordering of single-handed helical polymers. J. Am. Chem. Soc. 130, 229 (2008).

58 Nishimura, T., Tsuchiya, K., Ohsawa, S., Maeda, K., Yashima, E., Nakamura, Y. \& Nishimura, J. Macromolecular helicity induction on a poly(phenylacetylene) with $\mathrm{C}_{2}$ symmetric chiral [60]fullerene-bisadducts. J. Am. Chem. Soc. 126, 11711 (2004).

59 Kawauchi, T., Kumaki, J., Kitaura, A., Okoshi, K., Kusanagi, H., Kobayashi, K., Sugai, T., Shinohara, H. \& Yashima, E. Encapsulation of fullerenes in a helical PMMA cavity leading to a robust processable complex with a macromolecular helicity memory. Angew. Chem. Int. Ed. 47, 515 (2008) 
60 Miyagawa, T., Yamamoto, M., Muraki, R., Onouchi, H. \& Yashima, E. Supramolecular helical assembly of an achiral cyanine dye in an induced helical amphiphilic poly(phenylacetylene) interior in water. J. Am. Chem. Soc. 129, 3676 (2007).

61 Onouchi, H., Miyagawa, T., Morino, K. \& Yashima, E. Assisted formation of chiral porphyrin homoaggregates by an induced helical poly(phenylacetylene) template and their chiral memory. Angew. Chem. Int. Ed. 45, 2381 (2006).

62 Nishimura, T., Takatani, K., Sakurai, S. I., Maeda, K. \& Yashima, E. A helical array of pendant fullerenes on an optically active polyphenylacetylene. Angew. Chem. Int. Ed. 41, 3602 (2002).

63 Ohsawa, S., Maeda, K. \& Yashima, E. Syntheses and chiroptical properties of optically active helical poly(phenylacetylene)s bearing [60]fullerene pendants. Macromolecules 40, 9244 (2007).

64 Nishimura, T., Ohsawa, S., Maeda, K. \& Yashima, E. A helical array of pendant fullerenes on a helical poly(phenylacetylene) induced by non-covalent chiral interactions. Chem. Commun. 646 (2004).

65 Nonokawa, R. \& Yashima, E. Detection and amplification of a small enantiomeric imbalance in $\alpha$-amino acids by a helical poly(phenylacetylene) with crown ether pendants. J. Am. Chem. Soc. 125, 1278 (2003).

66 de Witte, P. A. J., Castriciano, M., Cornelissen, J. J. L. M., Scolaro, L. M., Nolte, R. J. M. \& Rowan, A. E. Helical polymer-anchored porphyrin nanorods. Chem. Eur. J. 9, 1775 (2003)

67 Maeda, K., Mochizuki, H., Watanabe, M. \& Yashima, E. Switching of macromolecular helicity of optically active poly(phenylacetylene)s bearing cyclodextrin pendants induced by various external stimuli. J. Am. Chem. Soc. 128, 7639 (2006).

68 Smith, D. K. Lost in translation? Chirality effects in the self-assembly of nanostructured gel-phase materials. Chem. Soc. Rev. 38, 684 (2009).

69 Goto, H., Zhang, H. Q. \& Yashima, E. Chiral stimuli-responsive gels: Helicity induction in poly(phenylacetylene) gels bearing a carboxyl group with chiral amines. J. Am. Chem. Soc. 125, 2516 (2003).

70 Hirst, A. R., Smith, D. K., Feiters, M. C. \& Geurts, H. P. M. Two component dendritic gel: effect of stereochemistry on the on the supramolecular chiral assembly. Chem. Eur. J. 10, 5901 (2004).

71 Hirst, A. R., Huang, B. Q., Castelletto, V., Hamley, I. W. \& Smith, D. K. Self-organisation in the assembly of gels from mixtures of different dendritic peptide building blocks. Chem. Eur. J. 13, 2180 (2007)

72 Morino, K., Oobo, M. \& Yashima, E. Helicity induction in a poly(phenylacetylene) bearing Aza-18-crown- 6 ether pendants with optically active bis(amino acid)s and its chiral stimuli-responsive gelation. Macromolecules 38, 3461 (2005).

73 Patra, T., Pal, A. \& Dey, J. Smart supramolecular hydrogel of $\mathrm{N} \alpha$-(4-n-Alkyloxybenzoyl)I-histidine exhibiting pH-modulated properties. Langmuir 26, 7761 (2010).

74 Luo, Z. L., Wang, S. K. \& Zhang, S. G. Fabrication of self-assembling d-form peptide nanofiber scaffold d-EAK16 for rapid hemostasis. Biomaterials 32, 2013 (2011).
75 Yao, X., Song, Y. L. \& Jiang, L. Applications of bio-inspired special wettable surfaces. Adv. Mater. 23, 719 (2011).

76 Qing, G. \& Sun, T. Chirality-triggered wettability switching on a smart polymer surface. Adv. Mater. 23, 1615 (2011).

77 Tomar, S., Green, M. M. \& Day, L. A. DNA-protein interactions as the source of largelength-scale chirality evident in the liquid crystal behavior of filamentous bacteriophages. J. Am. Chem. Soc. 129, 3367 (2007)

78 Barry, E., Beller, D. \& Dogic, Z. A model liquid crystalline system based on rodlike viruses with variable chirality and persistence length. Soft Matter 5, 2563 (2009).

79 Chung, W. J., Oh, J. W., Kwak, K., Lee, B. Y., Meyer, J., Wang, E., Hexemer, A. \& Lee, S. W. Biomimetic self-templating supramolecular structures. Nature 478, 364 (2011).

80 Geva, M., Frolow, F., Eisenstein, M. \& Addadi, L. Antibody recognition of chiral surfaces. enantiomorphous crystals of leucine-leucine-tyrosine. J. Am. Chem. Soc. 125, 696 (2003).

81 Geva, M., Eisenstein, M. \& Addadi, L. Antibody recognition of chiral surfaces. Structural models of antibody complexes with leucine-leucine-tyrosine crystal surfaces. Proteins 55, 862 (2004).

82 Sun, T., Han, D., Riehemann, K., Chi, L. \& Fuchs, H. Stereospecific interaction between immune cells and chiral surfaces. J. Am. Chem. Soc. 129, 1496 (2007).

83 Tang, K., Gan, H., Li, Y., Chi, L., Sun, T. \& Fuchs, H. Stereoselective interaction between DNA and chiral surfaces. J. Am. Chem. Soc. 130, 11284 (2008).

84 Gan, H., Tang, K., Sun, T., Hirtz, M., Li, Y., Chi, L., Butz, S. \& Fuchs, H. Selective adsorption of DNA on chiral surfaces: supercoiled or relaxed conformation. Angew. Chem. Int. Ed. 48, 5282 (2009).

85 Li, Y., Zhou, Y., Wang, H. Y., Perrett, S., Zhao, Y., Tang, Z. \& Nie, G. Chirality of glutathione surface coating affects the cytotoxicity of quantum dots. Angew. Chem. Int. Ed. 50, 5860 (2011).

86 Wang, X., Gan, H., Sun, T., Su, B., Fuchs, H., Vestweber, D. \& Butz, S. Stereochemistry triggered differential cell behaviours on chiral polymer surfaces. Soft Matter 6, 3851 (2010).

87 Wang, X., Gan, H. \& Sun, T. Chiral design for polymeric biointerface: the influence of surface chirality on protein adsorption. Adv. Funct. Mater. 21, 3276 (2011).

88 Yi, Q., Wen, X., Li, L., He, B., Nie, Y., Wu, Y., Zhang, Z. \& Gu, Z. The chiral effects on the responses of osteoblastic cells to the polymeric substrates. Euro. Polym. J. 45, 1970 (2009).

This work is licensed under the Creative Commons Attribution-NonCommercial-No Derivative Works 3.0 Unported License. To view a copy of this license, visit http:// creativecommons.org/licenses/by-nc-nd/3.0/

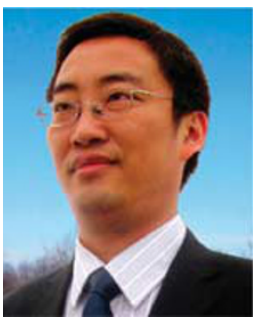

Professor Taolei Sun received his PhD from the Chinese Academy of Science (CAS) in 2002. After 2 years of postdoctoral research at the Institute of Chemistry, CAS, he worked at the Physics Institute of Muenster University, Germany, as an Alexander Von Humboldt Fellow. In 2006, he was awarded the Sofja Kovalevskaja by Alexander von Humboldt. Foundation and received funding support from the Federal Ministry of Education and Research of Germany to build an independent Bio and Nano-Interface research group in Germany. In late 2009, he joined Wuhan University of Technology, China, and was appointed as the "Chang-Jiang Scholar" distinguished professor. He works primarily on functional biointerface materials.

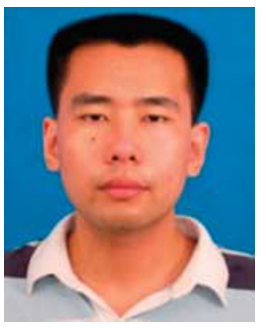

Professor Guangyan Qing received his PhD in 2008 from the College of Chemistry and Molecular Sciences at Wuhan University. In 2007, he joined the Bio- and Nano-Interface group led by Professor Taolei Sun at the Physics Institute, Muenster University, as an exchange student. One year later, he continued his work in Sun's group, as a postdoctoral researcher. In 2011, he joined the State Key Laboratory of Advanced Technology for Materials Synthesis and Processing at Wuhan University of Technology as a senior researcher. His main research interests include chiral sensors and smart polymer interface materials. 